\title{
Evaluación de la hemodiafiltración con reinfusión endógena en el tratamiento del riñón del mieloma
}

\author{
Victoria Eugenia Gómez López, Margarita Ruíz García, Carmen Lacalle Cano, Rafael Casas Cuesta \\ UGC Nefrología. Unidad de hemodiálisis. Hospital Universitario Reina Sofía. Córdoba
}

\section{Resumen}

Introducción: El mieloma múltiple ( $M M)$ es una enfermedad hematológica que produce un fracaso renal agudo (FRA) subsidiario de hemodiálisis en el $10 \%$ de casos.

Este fracaso renal, denominado riñón del mieloma, está producido por una nefropatía por cilindros, formados fundamentalmente por cadenas ligeras libres (CLL), que originan una obstrucción tubular que si no remite precozmente, produce daños glomerulares e insuficiencia renal crónica.

El tratamiento del riñón del mieloma se centra en reducir la formación de CLL con quimioterapia, y en eliminar las cadenas existentes reduciendo su concentración en plasma.

Las técnicas empleadas tradicionalmente en la depuración de CLL, utilizan membranas de muy alta permeabilidad, y presentan el inconveniente de la elevada pérdida de albúmina.

Recientemente ha aparecido la hemodiafiltración con reinfusión endógena (HFR), técnica que combina difusión, convección y adsorción.

Con esta técnica se utiliza una membrana de muy alta permeabilidad para la extracción de ultrafiltrado, y podría ser utilizada para la eliminación de CLL, salvando el inconveniente de la pérdida de albúmina que tienen otras técnicas.

Objetivo: Analizar la eliminación de CLL con esta técnica y su relación con la recuperación de la función renal, y el comportamiento de la albúmina sérica en tres pacientes tratados por FRA por MM.

\begin{tabular}{|c|}
\hline Correspondencia: \\
Victoria Eugenia Gómez López \\
UGC Nefrología, HU Reina Sofía \\
Avd. Menéndez Pidal s/n \\
14004. Córdoba \\
E-mail: rafcasas@ono.com
\end{tabular}

Pacientes y método: Estudiamos tres pacientes con edad media de 64 años tratados con HFR por FRA por MM.

Los tres fueron tratados con quimioterapia y HFR para la eliminación de CLL utilizando monitores fórmula ${ }^{T M}$ 2000, y Kit Supra 17 de Bellco®.

El tratamiento fue personalizado en función de los niveles de CLL, manteniéndose mientras el paciente requirió hemodiálisis.

Para valorar la eliminación de CLL se analizaron muestras de sangre y ultrafiltrado semanalmente.

Se determinó concentración de CLL y albúmina en sangre antes y después de la sesión, calculándose el porcentaje de reducción.

En ultrafiltrado se determinó concentración de CLL y albúmina antes y después de su paso por la resina adsortiva, al principio y final de la sesión.

Resultados: Se hicieron en total 30,59 y 66 sesiones, obteniéndose tasas de reducción media de CLL en plasma del $63,53.8$ y $38 \%$ respectivamente, sin modificaciones en los niveles de albúmina.

En ultrafiltrado, se obtuvieron tasas de reducción del 98, 99 y $99 \%$ al inicio de la sesión, y del 79, 58 y $72 \%$ al finalizar, sin modificaciones en los niveles de albúmina al pasar por la resina.

Solo uno de los pacientes recuperó función renal, al coincidir la instauración precoz del tratamiento y buena respuesta al tratamiento quimioterápico, no dándose estas circunstancias en los otros dos casos.

Conclusión: Los resultados indican que la técnica HFR elimina las CLL del plasma y mantiene la eliminación durante la sesión sin saturarse la resina, y sin pérdida de albúmina.

Con las lógicas reservas, por los pocos casos estudiados, podemos concluir que la HFR es una técnica que consigue una reducción importante y mantenida de las CLL en el MM con FRA, sin pérdida de albúmina, y que utilizada precozmente contribuye a la recuperación de la función renal en estos pacientes. 
PALABRAS CLAVE

- MIELOMA MÚLTIPLE

- CADENAS LIGERAS LIBRES

- HEMODIAFILTRACIÓN

- REGENERACIÓN DE ULTRAFILTRADO

\section{Evaluation of hemodiafiltration with endogenous reinfusion in the treatment of myeloma kidney}

\begin{abstract}
Introduction: Multiple myeloma ( $M M)$ is a hematologic disease that causes acute renal failure (ARF), requiring hemodialysis $10 \%$ of cases.

This renal failure, called myeloma kidney, is caused by the formation of urinary casts in the renal tubules from free light chains (FLCs). The tubular obstruction if not treated early, causes glomerular damage and chronic kidney disease.

Treatment of myeloma kidney focuses on reducing the formation of FLCs with chemotherapy and the elimination of existing chains reducing its concentration in plasma.

Techniques traditionally used in the clearance of FLCs, using very high permeability membranes, and have the disadvantage of the high albumin loss. Recently hemodiafiltration with endogenous reinfusion (HFR) has emerged, a technique that combines diffusion, convection and adsorption.

This technique uses a high permeability membrane to extract ultrafiltrate, and could be used for eliminating FLCs, overcoming the albumin loss.
\end{abstract}

Aim. To analyze the elimination of FLCs with this technique and its relationship to the recovery of renal function, and monitoring of serum albumin in three patients treated by ARF due to MM.

Material and methods: Three patients with ARF by $M M$, with a mean age of 64 years and treated with HFR were studied.

All patients were treated with chemotherapy and HFR for the elimination of FLCs using formulaTM 2000 monitors and Kit Supra 17 from Bellco®. The treatment is personalized according to the levels of FLCs, maintained during hemodialysis.

To evaluate the elimination of FLCs, blood samples and ultrafiltrates of the week were analyzed.
Pre- and post- session FLCs and serum albumin concentrations were determined, calculating the reduction ratio.

In ultrafiltrates, FLCs and albumin concentrations before and after passing through the adsorptive resin, at the beginning and end of the session were determined.

Results: 30, 59 and 66 sessions were made, resulting in mean reduction rates of FLCs concentrations of 63, 53.8 and $38 \%$ respectively, without changes in the albumin levels.

Reduction rates of 98,99 and $99 \%$ at the beginning of the session were obtained. At the end rates were of 79,58 y $72 \%$, without changes in albumin levels when passing through the resin. Only one patient recovered renal function.

Conclusions: Evidence shows that the HFR technique eliminates the FLCs and maintains this elimination during the session without saturation of the resin and without albumin loss.

Despite the small sample, we can conclude that the HFR is a technique that achieves a significant and sustained reduction of FLCs in patients with AFR due to $\mathrm{MM}$ with no loss of albumin. Moreover, its early use contributes to the recovery of renal function in these patients.

\section{KEYWORDS}

- MULTIPLE MYELOMA

- FREE LIGHT CHAINS

- HEMODIAFILTRATION

- ULTRAFILTRATE REGENERATION

\section{Introducción}

El mieloma múltiple ( $\mathrm{MM}$ ) es una enfermedad hematológica que aunque afecta al riñón en el $40 \%$ de casos, solamente del 12 al $20 \%$ desarrolla un fracaso renal agudo (FRA), que necesitará hemodiálisis en el $10 \%$ de ellos ${ }^{(1,2)}$.

En el MM las células plasmáticas de la médula ósea, cuya función principal es la síntesis de inmunoglogulinas están en una concentración muy superior a la normalidad, y su actividad sintetizando las moléculas que forman las inmunoglobulinas está también aumentada.

Cada inmunoglobulina está formada por cuatro cadenas de aminoácidos unidas entre sí. Dos de ellas son cadenas pesadas, y otras dos ligeras que pueden ser del tipo Lambda o Kappa. 
La causa más frecuente del FRA en el MM es la producción excesiva de cadenas ligeras libres (CLL), que ocasionan nefropatía por cilindros conocida por riñón del mieloma.

Estos cilindros están formados por fragmentos celulares, CLL y otras proteínas, y precipitan en la nefrona distal produciendo una obstrucción tubular(3).

Las CLL kappa y lambda son moléculas con un tamaño de 22,5 y $45 \mathrm{KDa}$ respectivamente, y en la insuficiencia renal su vida media está aumentada, lo que hace que aumente la exposición del riñón a las mismas ${ }^{(4)}$.

El tratamiento del riñón del mieloma se orienta pues, a conseguir la reducción de la exposición del riñón a estas CLL. Esto se lleva a cabo actuando sobre el MM mediante tratamiento quimioterápico (dexametasona, bortezomid, ciclofosfamida, etc...) para reducir su producción, al mismo tiempo que se utilizan como tratamiento coadyuvante, técnicas de depuración extracorpórea para eliminarlas y reducir su concentración en plasma ${ }^{(3,5)}$.

La recuperación de la función renal dependerá no solo de la reducción de las CLL circulantes, sino también de la velocidad en la que se consigue esta reducción, tal y como ponen de manifiesto Hutchison et $\mathrm{al}^{(6)}$, que afirman que en los pacientes que se consigue una reducción sostenida de los niveles de CLL circulantes en las tres primeras semanas de tratamiento, tienen mayor probabilidad de recuperar la función renal.

El primer intento de eliminación de CLL del plasma, se Ilevó a cabo con plasmaféresis, pero aunque los estudios iniciales parecían prometedores, después se ha comprobado que la eliminación de CLL es escasa, debido a la distribución corporal de CLL ( $80 \%$ extravascular), y que al poseer los plasmafiltros un elevadísimo tamaño de poro, se pierden gran cantidad de otras proteínas esenciales ${ }^{(4)}$.

Otra modalidad de tratamiento con la que se han conseguido excelentes resultados ${ }^{(7,8,9)}$ es la hemodiálisis (HD) con membranas de diálisis de muy alta permeabilidad high-cut-off ( $\mathrm{HCO}$ ), con un tamaño de poro entre 45 y $60 \mathrm{KDa}$, diseñadas específicamente para el riñón del mieloma, aunque presenta importantes inconvenientes como la elevada pérdida de albúmina, sobre todo cuando se asocia a transporte convectivo, disminución de los niveles de quimioterápicos aal tener estos una alta tasa de unión a proteínas, y su elevado $\operatorname{coste} \mathrm{e}^{(5,10)}$.

Recientemente ha aparecido la hemodiafiltración con reinfusión endógena (HFR), técnica susceptible de ser utilizada en el tratamiento del riñón del mieloma para eliminar las CLL, al mismo tiempo que se realiza la terapia renal sustitutiva necesaria en estos pacientes con FRA.

Se trata de una técnica que combina la convección, difusión y adsorción. En ella se utiliza un dializador capilar de doble cámara. En la primera, fabricada con una membrana de muy alta permeabilidad con un tamaño de poro de $42 K D$, que permite el paso de las CLL sobre todo kappa, se realiza una ultrafiltración. Este ultrafiltrado se pasa por un cartucho de resina adsortiva en el que quedan toxinas unidas a proteínas y teóricamente las CLL, con la ventaja de que no se adsorbe la albúmina, y se infunde una vez regenerado, incorporándolo al circuito hemático entre las dos cámaras del dializador(11,12).

La segunda cámara del dializador, está fabricada con una membrana de baja permeabilidad, y en esta se realiza una HD convencional (figura $\mathbf{1}$ ).

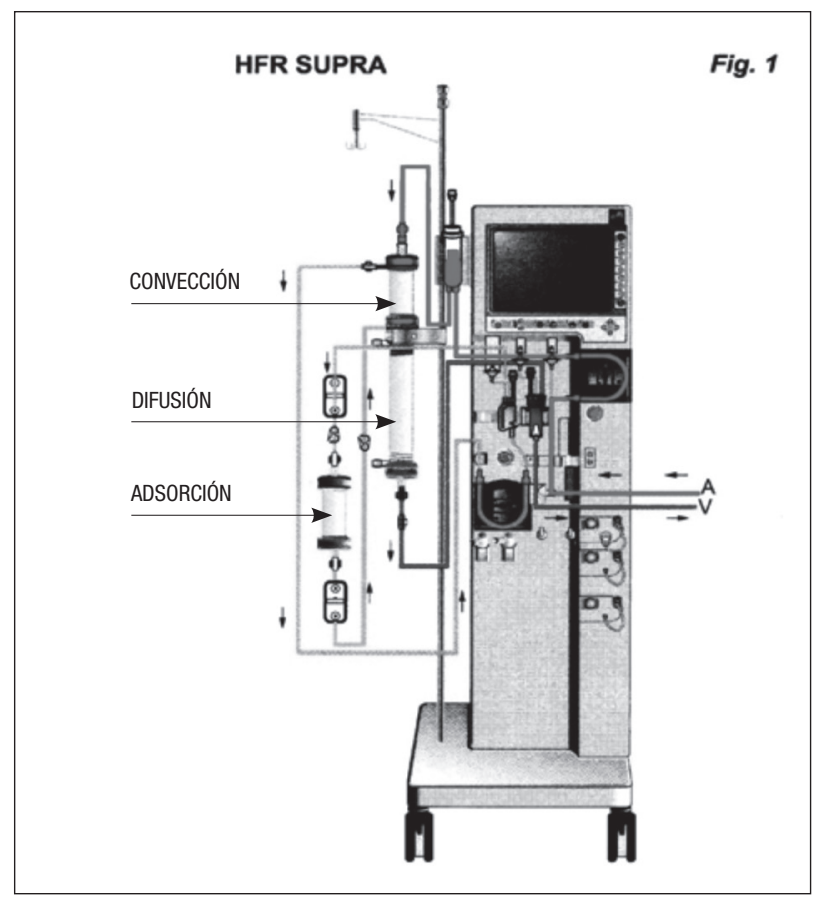

Figura 1

Al ser esta técnica de reciente utilización en el MM, no existen muchos resultados publicados al respecto, por lo que parece pertinente aportar nuestros datos aunque se trate de una serie muy corta.

El objetivo de este estudio fue analizar la eliminación de CLL y su relación con la recuperación de la función renal, y el comportamiento de la albúmina sérica en tres pacientes con MM y FRA subsidiarios de HD. 


\section{Material y método}

Se estudiaron tres pacientes (dos mujeres y 1 varón) con edad media de 64 años (52-79), y con FRA por MM diagnosticados y tratados en nuestro centro desde Julio de 2012 a Enero de 2013.

Dos de ellos no tenían antecedentes de afectación renal antes de la aparición del MM, mientras el otro padecía una insuficiencia renal crónica que se agudizó con el MM.

Los tres fueron tratados además de con agentes quimioterápicos, con HFR para la eliminación de CLL circulantes, utilizando monitores fórmula ${ }^{\mathrm{TM}} 2000$ y kit específico para esta técnica KIT SUPRA 17de (Bellco ${ }^{\circledR}$ ).

La pauta de tratamiento (número de sesiones y duración) se ajustó de forma personalizada en función de los niveles plasmáticos de CLL.

El flujo de sangre utilizado osciló entre 300 y $350 \mathrm{ml} / \mathrm{min}$, el de líquido de diálisis se fijó en $500 \mathrm{ml} / \mathrm{min}$, y el flujo de ultrafiltrado para su regeneración y posterior reinfusión, se fijó automáticamente por el programa del monitor. La pérdida de peso se programó en función de la ganancia de peso interdiálisis del paciente.

El tratamiento con HFR se decidió mantener mientras el paciente requiriera diálisis, aún cuando los niveles de CLL circulantes estuvieran por debajo de $500 \mathrm{mg} / \mathrm{l}$, ya que la $\mathrm{HFR}$ es ligeramente más cara que otras técnicas de hemodiafiltración, y no presenta mayores dificultades técnicas para su realización ${ }^{(13)}$.

Para estudiar la capacidad de eliminación de CLL con esta técnica, establecimos un protocolo de extracción de muestras sanguíneas y de ultrafiltrado según la siguiente pauta:

Extracción de muestras sanguíneas de la línea arterial pre y post sesión, y de ultrafiltrado pre y post resina a los 5 minutos de iniciar la sesión y 5 minutos antes de finalizarla.

Estas muestras (2 de sangre y 4 de ultrafiltrado), se extrajeron el primer día de diálisis de la semana. En ellas se determinó CLL y albúmina, y se calculó el porcentaje de reducción de sus niveles en plasma durante la sesión, y porcentaje de reducción de niveles de CLL y albúmina en el ultrafiltrado al pasar por la resina, al principio y al final de la sesión de HFR.

\section{Resultados}

Dos de los pacientes recibieron sesiones de HFR tres días por semana, con un total de 30 y 59 sesiones res- pectivamente, y el otro paciente recibió inicialmente sesiones diarias, disminuyendo el tiempo y frecuencia de las mismas según la función renal y niveles plasmáticos de CLL, realizándose en total 66 sesiones.

Respecto a la eliminación de CLL en plasma (Tabla 1), se obtuvieron tasas de reducción media por sesión del $63.4 \%, 53.8 \%$ y $38 \%$, mientras que los niveles de albúmina no se modificaron en dos pacientes y se elevaron discretamente en uno de ellos.

Tabla 1. Eliminación de CLL y Albúmina en suero.

\begin{tabular}{|l|c|c|c|}
\hline & Tipo CLL & $\begin{array}{c}\text { Reducción CLL } \\
\text { Pre-Post HFR }\end{array}$ & $\begin{array}{c}\text { [ ] Albúmina } \\
\text { Pre-Post HFR }\end{array}$ \\
\hline Caso 1 & Kappa & $63.4 \%$ & Sin modificación \\
\hline Caso 2 & Kappa & $53.8 \%$ & Elevación discreta post HFR \\
\hline Caso 3 & Lambda & $38 \%$ & Sin modificación \\
\hline
\end{tabular}

En el ultrafiltrado se obtuvieron mayores tasas de reducción media de CLL que en sangre (Tabla 2).

Tabla 2. Eliminación de CLL y Albúmina en ultrafiltrado.

\begin{tabular}{|l|l|c|c|c|c|} 
Tipo CLL & $\begin{array}{c}\text { Tasa reducción } \\
\text { CLL pre-post } \\
\text { adsorción, al } \\
\text { inicio de HFR }\end{array}$ & $\begin{array}{c}\text { Tasa reducción } \\
\text { CLL pre-post } \\
\text { adsorción, al } \\
\text { final de HFR }\end{array}$ & $\begin{array}{c}\text { [ ] Albúmina } \\
\text { Pre-post } \\
\text { adsorción, al } \\
\text { inicio de HFR }\end{array}$ & $\begin{array}{c}\text { [ ] Albúmina } \\
\text { Pre-post } \\
\text { adsorción, al } \\
\text { final de HFR }\end{array}$ \\
\hline Caso 1 & Kappa & $98 \%$ & $79 \%$ & Sin modificación & Sin modificación \\
\hline Caso 2 & Kappa & $99 \%$ & $58 \%$ & Sin modificación & Sin modificación \\
\hline Caso 3 & Lambda & $99 \%$ & $72 \%$ & Sin modificación & Sin modificación \\
\hline
\end{tabular}

Al inicio del procedimiento las tasas medias de eliminación fueron del $98 \%, 99 \%$ y $99 \%$, y cinco minutos antes de finalizar las sesiones de $79 \%, 58 \%$ y $72 \%$ respectivamente.

Los niveles de albúmina en el ultrafiltrado no sufrieron cambios al pasar por la resina, ni al inicio ni al final de las sesiones.

Solo un paciente que inició el tratamiento con HFR precozmente, recuperó función renal. A las tres semanas de tratamiento las cifras de CLL eran inferiores a $500 \mathrm{mg} / \mathrm{l}$ y la creatinina se situaba en torno a $3 \mathrm{mg} / \mathrm{dl}$. Se inició una reducción progresiva de sesiones de HFR y a los tres meses salió del programa de HD.

En otro paciente se instauró el tratamiento con HFR a los dos meses de detectarse el MM y además no respondió al tratamiento quimioterápico, falleciendo por shock séptico tras un autotrasplante de células hematopoyéticas. 
El tercer paciente tampoco recuperó función renal, al tratarse de un paciente con ERC reagudizada por el MM. A pesar de recibir tratamiento precozmente, no mejoró la función renal y permanece dependiente de HD.

\section{Discusión}

Nuestros resultados nos indican que la técnica HFR es capaz de eliminar las CLL del plasma y mantener la pérdida durante toda la sesión sin saturar la resina, sin que exista pérdida de albúmina.

Leung et al, en un trabajo de 2008 afirma que los pacientes con MM tratados con plasmaféresis y quimioterapia, cuando redujeron en más del $50 \%$ sus niveles de CLL en plasma, recuperaron en el $75 \%$ de casos la función renal $^{(14)}$.

En nuestro estudio hemos obtenido tasas de reducción superiores en los casos de MM Kappa y del 38\% en el MM lambda, a pesar de que las CLL lambda tienen un tamaño molecular superior al poro de la membrana utilizada en la cámara de ultrafiltración en la técnica HFR.

Otros estudios realizados con membranas $\mathrm{HCO}$ en combinación también con quimioterapia, consiguieron altas tasas de reducción de CLL tanto Kappa como lambda, logrando tasas de recuperación de función renal en torno al $60-74 \% \%^{(10,15,16)}$.

El resultado obtenido en nuestro estudio sobre el porcentaje de reducción de las CLL Lambda (38\%), no deja de Ilamarnos la atención al ser este un resultado no esperable, ya que el tamaño del poro de la membrana utilizada en la técnica HFR para la ultrafiltración, en sensiblemente inferior al de estas moléculas.

Pensamos que la eliminación de CLL lambda con esta técnica, aunque debe ser objeto de otro estudio, puede deberse al importante transporte convectivo que ocurre en la cámara de ultrafiltración. En esta cámara, que tiene una superficie pequeña $(0.7 \mathrm{~m} 2)$, se aplica un flujo de ultrafiltración proporcionalmente tan elevado o incluso más que en una hemodiafiltración convencional en las que se utilizan membranas con el triple de superficie.

Aun habiendo obtenido en nuestro estudio tasas de reducción de CLL Kappa, ligeramente inferiores a las que se obtienen con membranas $\mathrm{HCO}$, y sustancialmente inferiores en el caso de MM Lambda, el que sólo un paciente recuperara la función renal se puede explicar debido a las circunstancias que rodearon cada caso.

El caso que recuperó función renal, se trataba de un MM con CLL Kappa las cuales se eliminan en altas tasas con la HFR, la paciente tuvo buena respuesta al tratamiento quimioterápico, y se instauró el tratamiento precozmente. Estas circunstancias son las que se describían en el estudio de Hutchison el al. ${ }^{(6)}$, y Khalafallah et al. ${ }^{(7)}$, como circunstancias favorables para la recuperación de la función renal.

Otro de los casos fue un paciente que aunque se obtuvieron tasas de eliminación de CLL Kappa del 63\%, la paciente no respondió al tratamiento quimioterápico, y se instauró el tratamiento con HFR a los dos meses de diagnosticarse el MM, y el tercer caso además de ser un MM lambda en el que la HFR obtiene discretas tasas de eliminación de CLL, se trataba de un paciente con ERC reagudizada por el MM.

Otro de los parámetros estudiados y que puede suponer una ventaja de la HFR sobre la HD con membranas $\mathrm{HCO}$, es la albúmina sérica. Hemos encontrado que en la HFR no se modifica, frente a una pérdida considerable con las membranas $\mathrm{HCO}$, pérdidas que hay que reponer, suponiendo esto un coste adicional para esta técnica ya de por sí cara. De hecho se calcula que la sesión de HFR, cuesta entre ocho y nueve veces menos que una sesión con membrana $\mathrm{HCO}$, sin tener en cuenta los gastos de personal ${ }^{(17)}$.

Teniendo en cuenta las limitaciones de la HFR para la eliminación de CLL lambda, y las reservas que debemos tener por el escaso número de pacientes estudiado, podemos concluir que la HFR es una técnica de depuración extrarrenal que consigue una reducción importante y mantenida de las CLL Kappa en el MM con FRA, sin que se produzcan pérdidas de albúmina, y que utilizada precozmente contribuye a la recuperación de la función renal en estos pacientes.

\section{Conflicto de intereses}

Los autores declaran estar libres de cualquier asociación personal o comercial que puedan suponer un conflicto de interés relacionado con el contenido de este trabajo.

Recibido: 10 Agosto 2014

Revisado: 14 Agosto 2014

Modificado: 14 Agosto 2014

Aceptado: 14 Agosto 2014 


\section{Bibliografía:}

1. Kyle RA, Gertz MA, Witzing TE, Lust JA, Lacy MQ, Dispenzieri A, et al. Review of 1027 patients with newly diagnosed multiple mieloma. Mayo Clin Proc 2003; 78:21-33.

2. Knudsen $L M$, hjorth $M$, Hippe $E$. Renal failure in multiple myeloma: reversibitity and impact on prognosis. Eur J Haematol 2000; 65:175-81.

3. Isaac J, Herrera del Castillo G. El riñón en el mieloma y en las enfermedades de cadenas ligeras y pesadas, En: Hernando L, Aljama $P$, Arias $M$ (eds). Nefrología Clínica. 3 ed. Madrid: Panamericana; 2008. P. 450-60.

4. Álvarez de Lara MA, Martín Malo A, Aljama García P. Nuevas alternativas en el tratamiento del riñón del mieloma. Nefrología 2013; 33(4): 443-7.

5. Hutchison CA, Cockwell P, Reid S, Chandler K, Mead G, Harrison J, et al. Efficient removal of immunoglobulin free light chain by hemodialysis for multiple myeloma in vitro and in vivo studies. J Am Soc Nephrol 2007; 18: 886-95.

6. Hutchison CA, Cockwell $P$, Stringer $S$, Bradwell A, Cook M, Gertz MA, et al. Early reduction of serum-free light chains associates with renal recovery in myeloma kidney. J Am Soc Nephrol 2011; 22: 1129-36.

7. Khalafallah A, Wuong S, Love S, Mohamed M, Mace R, Khalil R, et al. Early application of High Cut-of Haemodialysis for de novo myeloma nephropathy is associated with long-term dialysis independency and renal recovery. Mediterr $\mathrm{J} \mathrm{He}$ matol Infect Dis 2013; 5: e2013007.

8. Basnayake $K$, Hutchison CA, Kamel D, Sheaff M, Ashman N, Cook M, et al. Resolution of a cast nephropathy following free light chain removal by haemodialysis in a patient with multiple myeloma: a case report. J Med Case Rep 2008; 2: 380.

9. Hutchison CA, Harding $S$, Mead G, Goehl H, Storr M, Bradwell $A$, et al. Serum free-light chain removal and supportive care. Artif Organs 2008; 32: 910-7.
10. Hutchison CA, Bradwell A, Cook M, Basnayake $K$, Basu S, Harding $S$, et al. Treatment of acute renal failure secondary to multiple myeloma with chemotherapy and extended high cutoff hemodialysis. Clin J Am Soc Nephrol 2009; 4: 745-54.

11. Testa A, Dejoie T, Lecarrer D, Wratten M, Sereni L, Renaux JL. Reduction of free immunoglobulin light chains usin adsorption properties of haemodiafiltration with endogenous reinfusion. Blood Purif 2010; 30: 34-6.

12. Pendón-Ruiz de Mier MV, Ojeda López R, Álvarez de Lara Sánchez MA, Martín Malo A, Aljama García P. Eficacia de la HFR SUPRA en la insuficiencia renal por mieloma múltiple. Nefrología 2013; 33(3): 426-8.

13. Ramirez Moreno $\mathrm{C}$, et al. Análisis comparativo entre hemodiafiltración en línea y hemodiafiltración con reinfusión endógena: parámetros clínico-técnicos e inflamatorios. Enferm. Nefrol. 2014; 17(2): 104-109.

14. Leung N, Gertz MA, Zelsenrust SR, Rajkumar SV, Dispenzieri A, Fervenza FC, et al. Improvement of cast nephropathy with plasma Exchange depends on the diagnosis and on reduction of serum free light chains. Kidney Int 2008; 73: 1282-8.

15. Hutchison CA, Heyne N, Airia P, Schindler R, Zickler $D$, Cook $M$, et al. Inmunoglobulin free light chain level and recovery from mieloma kidney on treatment with chemotherapy and high cut-off haemodialysis. Nephrol Dial Transplant 2012; 27 (10): 3823-8.

16. Martín-Reyes G, Toledo-Rojas R, Torres-Rueda A, Sola-Moyano E, Blanca-Martos L, FuentesSánchez $L$, et al. Tratamiento con hemodiálisis del fracaso renal agudo en el mieloma múltiple con filtros de alto poro (High Cut Off). Nefrología 2012; 32(1):35-43.

17. Pendón-Ruiz de Mier MV,Álvarez de Lara Sánchez MA, Ojeda López R, Martín Malo A, Carracedo J, Caballero Villarraso J, Corona A, Aljama García P. Eficacia de la hemodiafiltración con regeneración del ultrafiltrado en la reducción de cadenas ligeras en el mieloma múltiple con insuficiencia renal. Nefrología 2013 ; 33(6): 788-96. 